\title{
Meta
}

Journal des traducteurs

Translators' Journal

\section{Xu, J. (2003) : De la traduction, Wuhan, Éditions de l'Éducation du Hubei, Chine, 463 p.}

\section{Yunhong Liu}

Volume 52, numéro 3, septembre 2007

URI : https://id.erudit.org/iderudit/016742ar

DOI : https://doi.org/10.7202/016742ar

Aller au sommaire du numéro

Éditeur(s)

Les Presses de l'Université de Montréal

ISSN

0026-0452 (imprimé)

1492-1421 (numérique)

Découvrir la revue

Citer ce compte rendu

Liu, Y. (2007). Compte rendu de [Xu, J. (2003) : De la traduction, Wuhan,

Éditions de l'Éducation du Hubei, Chine, 463 p.] Meta, 52(3), 574-576.

https://doi.org/10.7202/016742ar

Ce document est protégé par la loi sur le droit d'auteur. L'utilisation des services d'Érudit (y compris la reproduction) est assujettie à sa politique d'utilisation que vous pouvez consulter en ligne.

https://apropos.erudit.org/fr/usagers/politique-dutilisation/
Cet article est diffusé et préservé par Érudit.

Érudit est un consortium interuniversitaire sans but lucratif composé de l'Université de Montréal, l'Université Laval et l'Université du Québec à Montréal. Il a pour mission la promotion et la valorisation de la recherche. https://www.erudit.org/fr/ 
All twenty-one papers in The Critical Link 3 help us realize that community interpreting truly lies at the heart of the everyday lives of a great number of people around the world. In many situations including healthcare, administration and legal services, interpreters are the "critical links" that enable communities to function properly. Despite the impact that community interpreters have on societies, unfortunately, so far, interpreting studies have not given enough attention to this area of interpreting. Rather than take issues of interpreting quality and interpreter qualifications as an excuse for lesser academic interest, efforts should be made to achieve a real understanding of what community interpreting entails and to come up with specific measures that can support the substantial development of its many dimensions.

In this endeavor, The Critical Link 3 will definitely be a helpful map that guides the footsteps of anyone who plans to traverse the world of community interpreting.

Jungwha Choi

Hankuk University of Foreign Studies, Seoul, Korea

Xu, J. (2003): De la traduction, Wuhan, Éditions de l'Éducation du Hubei, Chine, $463 \mathrm{p}$.

De la traduction cherche à étudier les problèmes fondamentaux qui se posent aux théoriciens comme aux praticiens de la traduction malgré le temps et l'espace, et qui se perpétuent tout le long de son histoire. Cet ouvrage est la cristallisation d'une trentaine d'années de recherches et de réflexions inlassables effectuées par le professeur Xu sur l'acte de traduire. Rappelons qu'avant cette présente publication, il a déjà fait publier plusieurs ouvrages dans lesquels il a suggéré la classification de la traduction en analysant les éléments interdépendants et interactifs de ses trois niveaux: niveau de pensée, niveau sémantique et niveau esthétique, et a explicité les problèmes posés à la traduction en les situant sous trois aspects: volonté, réalité et rationalité. Composé de 7 chapitres avec une longue introduction et une conclusion, De la traduction se présente donc comme le prolongement des études antérieures qui constituent une base solide pour la rédaction de cette œuvre énormément fructueuse. Après la publication, cet ouvrage riche en valeurs théoriques a suscité une grande réaction de la part des traductologues, des traducteurs, des écrivains et des critiques chinois. Une dizaine de critiques et d'études lui ont été consacrées depuis un an. Le premier tirage (6000 exemplaires) est presque épuisé.

Dans le premier chapitre, le professeur Xu médite la nature de la traduction, la plus essentielle problématique ontologique, et tente de répondre à la question suivante: Qu'est-ce que la traduction? C'est une question qui se pose sans délai quand la traduction, activité fort ancienne en Chine comme en Occident, fait objet des réflexions systématiques. Mais la réponse paraît très difficile à formuler, car on peut constater qu'il existe nombre de définitions données au mot «traduction» qui s'avèrent aussi complexes que diverses. Après avoir exploré les compréhensions et les conceptions de l'activité traduisante qui se diversifient selon qu'elles sont de différentes disciplines et de différentes époques, en mettant en valeur cinq caractéristiques essentielles de la traduction telles que socialité, nature culturelle, transformation des signes, créativité et historicité, l'auteur essaie de définir la traduction comme l'activité de communication transculturelle qui s'effectue par la transformation des signes et qui a pour tâche la reproduction du sens.

Le deuxième chapitre aborde une explicitation du processus de la traduction. On a déjà remarqué que la traduction devait être comprise non seulement comme un résultat statique, mais encore et surtout comme un processus dynamique, qui implique qu'elle se situe dans un système constitué de façon interdépendante par l'œuvre originale et son auteur, l'œuvre 
traduite et son traducteur, et enfin le lecteur. D'après M. Lederer et D. Seleskovitch, le processus de la traduction peut être divisé en quatre phases: compréhension, déverbalisation, réexpression et vérification. George Steiner distingue quatre phases également, mais quelque peu différentes: élan de confiance, agression, incorporation et restitution. Jean-René Ladmiral, lui, le divise en deux phases «bien distinctes» : lecture-interprétation et réécriture. Quelles que soient la complexité du processus de la traduction et la diversité d'idées des théoriciens, traduire, c'est d'abord comprendre. Ainsi, se basant sur l'herméneutique moderne, le professeur Xu souligne la nécessité de prendre en considération la créativité du sujet traduisant ainsi que les possibilités et les limites de l'interprétation. De plus, en faisant des réflexions sur l'historicité de la compréhension et donc de la traduction, il traite le phénomène de la retraduction et démontre que la raison d'être et la nécessité de la retraduction résident précisément dans le fait que la traduction a pour tâche d'assurer le prolongement et l'élargissement de la vie de l'œuvre d'origine.

Dans le troisième chapitre, après l'élucidation de la tâche radicale de la traduction, le professeur Xu montre qu'il est nécessaire de considérer l'étude du sens comme un des volets primordiaux de la traductologie. Qu'il s'agisse de la traduction entre langues ou de la traduction entre signes, le sens joue toujours le rôle d'un axe dans tout le processus de la traduction, malgré des circonstances de production et de réception différentes, autrement dit, c'est le sens qui est l'enjeu de la traduction. À la suite de cette mise en valeur de l'importance du problème de sens, le professeur Xu compare la conception linguistique traditionnelle et celle de F. de Saussure et ainsi aboutit à remettre en question l'objectivité et la détermination du sens. Le sens ne peut être dès l'abord révélé, ce n'est que dans la lecture qui comprend et la communication authentique que se réalise sa concrétisation.

Le quatrième chapitre se présente comme une analyse des éléments qui peuvent exercer des influences sur la traduction et même y faire obstacle. Le processus de la traduction n'est certainement pas un processus clos, tout le parcours de l'activité traduisante, de la sélection de l'œuvre originale à traduire jusqu'à la réception et à la diffusion de l'œuvre traduite dans la langue d'arrivée, est plus ou moins conditionné par différents éléments tels que conditions sociales, valeurs culturelles, attente esthétique du lecteur, etc., qui ne sont jamais stables et qui se montrent complètement ouverts et changeables en fonction des époques. Après cette prise de position, le professeur Xu explicite de façon systématique huit éléments essentiels qui entrent en jeu dans l'activité traduisante: contexte culturel et facteurs sociaux, idéologie et facteurs politiques, motif de la traduction et conception de la traduction, relations langagières et compétence du traducteur. À travers la révélation de ces obstacles à la traduction, la poursuite aveugle de la fidélité absolue au texte original deviendrait la conscience plus rationnelle des possibilités et des impossibilités de la traduction, ce qui nous aiderait mieux à aboutir à la finalité de la traduction.

Dans le quatrième chapitre, on voit que la traduction constitue un processus complexe qui est conditionné par de nombreux éléments linguistiques et extra-linguistiques; il s'ensuit que de nombreuses dichotomis conceptuelles telles que traduction littérale / traduction libre, traduction des mots / traduction du sens, fidélité à la forme / fidélité au fond, fidélité au corps / fidélité à l'âme, imitation / recréation, fidélité / trahison, exotisme / naturalisation, traduction sourcière / traduction cibliste, traduisibilité / intraduisibilité, etc. viennent perturber le dévoilement de l'acte de traduire. En fait, toutes ces dichotomies aussi complexes que diversifiées dérivent de trois oppositions dualistes fondamentales: traduisibilité / intraduisibilité, étranger / même, corps / âme. Ainsi, dans le cinquième chapitre, la discussion est centrée sur ces trois dichotomies clé de la traduction, constatées tout le long de son histoire. Après les avoir élucidées à fond en parcourant les points de vue de bien des traducteurs chinois et étrangers en cette matière, le professeur Xu insiste sur la nécessité d'avoir avant tout une conception nette de la traduction pour se débarrasser de tous les dualismes conventionnels qui tourmentent les traducteurs ainsi que les traductologues. 
Dans le sixième chapitre, l'auteur explore le rôle joué par le sujet traduisant dans l'acte de traduire et l'interaction entre l'auteur, le traducteur et le lecteur. Rappelons que, dans Pour une critique des traductions: John Donne, Antoine Berman propose fermement qu'il faudrait «aller au traducteur» et le considère comme un tournant méthodologique d'autant plus essentiel que l'une des tâches d'une herméneutique du traduire est précisément la prise en vue du sujet traduisant. En appréciant cette prise de conscience, le professeur Xu se pose immédiatement la question suivante: Qui est le traducteur? et suggère la réhabilitation du traducteur. Quelle est l'image du traducteur pour l'auteur, le lecteur, le critique, ou pour le traducteurs lui-même? «Imitateur», «traitre», " esclave», «transmetteur du sens», "passeur des mots», «messager culturel», etc., ces appellations, favorables ou défavorables (pour la plupart), justes ou partiales, peuvent-elles évoquer une image authentique du traducteur? Il paraît qu'on ne peut aborder le problème du sujet traduisant sans évoquer le fameux adage italien Traduttore tradittore, qui continue à être un jugement définitif de la traduction. En remettant en cause l'identité traditionnelle du traducteur, l'auteur ébranle l'éternelle opposition absolue entre fidélité et trahison ou fidélité et recréation, explicite de façon dialectique qu'il faudrait attacher de l'importance à la subjectivité, à l'interaction entre l'auteur, le traducteur et le lecteur pour reconstituer la conception de la traduction allant de l'opposition à la complémentarité, du monologue au dialogue. En tant que produit spirituel et non absolument indépendant, la traduction doit posséder des éléments recréatifs, mais cette recréation a son degré inhérent et limité, c'est pour cela que la traduction est le fruit d'une intersubjectivité authentique.

Le dernier chapitre est consacré à la critique de la traduction, côté relativement faible de la traductologie. Aucune traduction ne peut se passer de la critique, rien n'est plus regrettable pour un traducteur que de se heurter à l'absence de la critique et au silence du public. La critique de la traduction s'avère fatale et nécessaire pour la traduction, ce qui a été reconnu aujourd'hui par les traducteurs et les théoriciens. «Fatale», parce que nombre de textes critiques ne tardent pas à se faire lire et à assumer une tâche consistant à examiner les traductions pour en faire la revue, pour les élucider, les évaluer; «nécessaire», parce que la critique de la traduction devrait être influente et efficace pour la traduction, pour sa perfection et pour le prolongement de sa vie. Pourtant, ce qu' «elle devrait être» n'implique pas forcément ce qu' « elle est». D’où le souci de l'auteur: comment la critique de la traduction pourrait-elle se présenter comme une vraie critique faisant preuve d'une directivité et d'un esprit constructif? Après avoir montré la défectuosité des deux tendances néfastes constatées dans le milieu de la critique de la traduction - la première réside justement dans la comparaison simple et directe entre le texte original et sa traduction ou entre les diverses versions de traduction au niveau sémantique; la seconde réside dans les analyses globalistes visant à obtenir immédiatement un jugement définitif qui, en vérité, est basé seulement sur l'impression subjective du critique -, le professeur Xu propose d'élucider toutes les valeurs de la traduction sociale, culturelle, langagière, créative et historique et de situer ainsi la critique de la traduction dans un champ plus étendu pour qu'elle assume véritablement sa tâche de perfectionner, d'illustrer et de perpétuer sans cesse la traduction.

Nous n'avons pas analysé la totalité de l'ouvrage, mais nous tenons à en recommander la lecture à tous ceux qui s'intéressent aux approches et aux théories de la traduction, car les problèmes fondamentaux concernant la traduction y sont exposés et démontrés de manière dialectique et systématique avec une combinaison parfaite de la théorie et de la pratique, de l'héritage et de l'innovation. C'est une lecture indispensable pour mieux se dégager des conceptions conventionnelles de la traduction.

YUNHONG LIU Université de Nanjing, Nanjing, Chine 